\title{
The Upper Bound of Metric Dimension of Bridge Graphs
}

\author{
Amrullah Amrullah* \\ Mathematics Education Study Program \\ Universitas Mataram \\ Mataram, Indonesia \\ amrullah@unram.ac.id
}

\author{
Baidowi Baidowi \\ Mathematics Education Study Program \\ Universitas Mataram \\ Mataram, Indonesia \\ baidowiunram@gmail.com
}

\author{
Syahrul Azmi \\ Mathematics Education Study Program \\ Universitas Mataram \\ Mataram, Indonesia \\ syahrulazmi.fkip@unram.ac.id
}

\author{
Nani Kurniati \\ Mathematics Education Study Program \\ Universitas Mataram \\ Mataram, Indonesia \\ nanikurniati.fkip@unram.ac.id
}

\author{
. Turmuzi Turmuzi \\ Mathematics Education Study Program \\ Universitas Mataram \\ Mataram, Indonesia \\ mturmuzi@unram.ac.id
}

\begin{abstract}
The metric dimension is one of the problems in graph theory which is interesting to study until now. The metric dimension has many applications such as image processing. However, not all graphs have been obtained for the metric dimensions such as a bridge graph. A bridge graph is a graph obtained from two connected graphs $\boldsymbol{G}_{\mathbf{1}}, \boldsymbol{G}_{\mathbf{2}}$ with adding a new edge $\boldsymbol{u} \boldsymbol{v}$ where $\boldsymbol{u}$ in $\boldsymbol{G}_{\mathbf{1}}$ dan $\boldsymbol{v}$ in $\boldsymbol{G}_{\mathbf{2}}$. A bridge graph is a new graph which has a larger order than the previous graph $\boldsymbol{G}_{\mathbf{1}}$ or $\boldsymbol{G}_{\mathbf{2}}$. Therefore, the subdivision graph metric dimension can be obtained from the metric dimension before subdivision. Metric dimension of bridge graph can be determined by analysis of structure and analysis of the distance between vertices. This article shows the upper bound of a bridge graph from two connected graphs. In addition, this paper shows metric dimension the bridge graphs which obtained from the caterpillar and cycle graph.
\end{abstract}

Keywords - bridge graph, metric dimension, caterpillar, cycle graph.

\section{INTRODUCTION}

The metric dimension is one of the concepts of graph dimensions that has many applications. The application of the concept of metric dimensions appears in several papers such as robot navigation [1]-[3] and optimization of the placement of threat detection sensors [4]. The application of this concept cannot be used on all graphs because many graphs still have unknown dimension, such as bridge graphs. A bridge graph is a graph obtained from the operation of adding new sides of two graphs. This bridge graph is an interesting graph caused by the addition of orders from order of two graphs before operating with the bridge edge. Therefore, the metric dimension of a bridge graph can be determined by the metric dimensions of the two graphs before it is operated.

The dimensions of the graph such as metric and partition dimensions have been studied by several researchers. The metric dimension has been published in several articles as in [2], [3], [5]. Even the metric dimensions in certain class graphs are also researched, such as [6] giving metric dimensions of bipartite graphs, [7] metric dimensions from lexicographic products of graphs. As recently as 2019, [4] provided the metric dimension results from general wheels.
A bridge graph is a graph obtained from the operation of two graphs by adding one edge on the two graphs. Therefore, this bridge graph can be considered as the extend graph of the two previous graphs. In other words, if two graphs of smaller orders have been obtained the metric dimension, then the bridge operation can give the metric dimension of the bridge graph of the larger order. Therefore, the metric dimension bridge graph is one of the interesting graphs in dimension graph research. Although, until now the research of metric dimension on bridge graphs has not published it yet. However, the other variants of the metric dimension, namely the partition dimension have been published such as the partition dimension od subdivision on the star graph [8]. [9] the partition dimension of subdivision homogeneous caterpillar [10], homogeneous firecrackers [11], subdivision of a Complete graph [8], [12].

The problems in this article are to find the metric dimension of the graphs especial the bridge graphs. This paper shows the upper bound the metric dimensions of bridge graphs from two connected graphs. The bridge graph is a graph that is obtained from two graphs connected by two vertices of the two graphs in the form of edges. We show also the metric dimension the bridge graph form cycle $\mathrm{C} \_\mathrm{m}$ and a homogeneous caterpillar $\mathrm{C}(\mathrm{m}, \mathrm{n})$. Let $\mathrm{G}$ be a connected graph, and $x, y \in V(G)$. The distance between the vertex $x$ and $y$ in $G, d(x, y)$, is the length of a shortest path from $x$ to $y$. Let $\mathrm{W}=\left\{\mathrm{w} \_1, \mathrm{w} \_2, \cdots, \mathrm{w} \_\mathrm{k}\right\} \subset \mathrm{V}(\mathrm{G})$. The representationof $\mathrm{x}$ respect to $\overline{\mathrm{W}}, \mathrm{r}(\mathrm{x} \mid \mathrm{W})=\left(\mathrm{d}\left(\mathrm{x}, \mathrm{W} \_1\right), \mathrm{d}\left(\mathrm{x}, \mathrm{w} \_1\right), \cdots, \mathrm{d}\left(\mathrm{x}, \mathrm{w} \_\mathrm{k}\right)\right)$. If $\mathrm{r}(\mathrm{x} \mid \mathrm{W}) \neq \mathrm{r}(\mathrm{y} \mid \mathrm{W})$ for $\mathrm{x} \neq \mathrm{y} \in \mathrm{V}(\mathrm{G})$, then $\mathrm{W}$ is called a resolving set of $G$. The metric dimension of $G, \operatorname{dim}(G)$, is a minimum cardinality of all resolving set of $\mathrm{G}$.

\section{METHOD}

The research is focused on determining of metric dimensions of the bridge graphs on caterpillar and cycle graphs. We begin by determining the uppers bound of the metric dimension of bridge graphs of any connected graph and the cycle graph. Next, the research is continued to determine exactly the metric dimensions of the specific graphs namely caterpillar and cycle graphs. Furthermore, this research was carried out in several stages as follows. 
We determine the upper bound of the metric dimension of the component of a connected graph $G$. Let $G_{1}$ and $G_{2}$ be two connected graphs and $u \in V\left(G_{1}\right), v \in V\left(G_{2}\right)$. Determination of the upper bound $\operatorname{dim}\left(B\left(G_{1}, G_{2}, u v\right)\right)$ used the properties of degree the vertices $u$ and $v$.

Determination of the upper bound of the metric dimension of the bridge graph used properties of component the bridge graph.

We determine the exact value of metric dimension on specific homogeneous caterpillar graph $C_{m, n}$, and cycle graph $C_{p}$. We explore the properties of bridge graph which considers the following properties.

Properties of the pendant and non-pendant edge of homogeneous caterpillar graph. Let $u v$ be an edge of graph $G$. The edge $u v$ is called the pendant edge if one of vertices $\{u, v\}$ is degree one, and the otherwise is called non-pendant edge

Properties of the vertex on cycle graph which is connected to a vertex of the homogeneous caterpillar graph.

Properties of distance two vertices $u$ and $v$ to any vertex $x$ in bridge graph $B\left(G_{1}, G_{2}, u v\right)$ such that $d(u, x) \neq d(v, x)$ for any $x \in V\left(B\left(G_{1}, G_{2}, u v\right)\right)$.

\section{RESULTS AND DISCUSSION}

In this section, we show the upper bound of the metric dimension from the components which is obtained from a graph that is removed the bridge edge. Let $G_{1}$ and $G_{2}$ be two connected graphs, $a \in V\left(G_{1}\right), b \in V\left(G_{2}\right)$. The bridge graph $B\left(G_{1}, G_{2}, a b\right)$ is a graph which is obtained from $G_{1}$ dan $G_{2}$ with lingking $a \in V\left(G_{1}\right)$ to $b \in V\left(G_{2}\right)[13]$.

\section{Theorem 1.}

Let $G$ be a connected graph and $u v$ be a bridge edge of $G, \operatorname{deg}(u) \geq 3, \operatorname{deg}(v) \geq 3$. Let $G_{1}$ and $G_{2}$ be two components of $G$ which are obtained by removing edge $u v$ of $G$. If $\operatorname{dim}(G)=k$ then $\operatorname{dim}\left(G_{i}\right) \leq k$ with $i \in\{1,2\}$.

\section{Proof}

For a contradiction, suppose $\operatorname{dim}\left(G_{i}\right) \geq k+1$. This means that there is a subset $W=\left\{w_{1}, w_{2}, \cdots, w_{k}, w_{k+1}\right\}$ as a basis of $G_{i}$.

We will show that $\operatorname{dim}\left(G_{i}\right) \leq k$ for $i \in\{1,2\}$. Let $x, y$ be two distinct vertices of $G_{i}$. Now consider endpoint $u$ of the bridge $u v$ in $G$. If $d(x, u)=d(y, u)$ then the vertices $x, y$ cannot be distinguished by any vertex in $\{u\} \cup V\left(G_{2}\right)$. Since $\operatorname{dim}(G)=k$, the vertices $x, y$ are distinguished by $t$ vertices $\left\{w_{1}, w_{2}, \cdots, w_{t}\right\}$ with $t<k$ of $V\left(G_{1}\right)$.

Next, we will show that $t \neq k$, suppose $t=k$. Since $\operatorname{deg}(v) \geq 3$, there is at least two vertices $x, y$ in $G_{2}$ which have the same distance to any vertex in $\{u\} \cup$ $V\left(G_{1}\right)$. Therefore, we have the vertices $x, y$ which cannot be distinguished by any vertex in di $\{u\} \cup V\left(G_{1}\right)$, a contradiction to $\operatorname{dim}(G)=k$. As a consequence, we obtain $t<k$. Let $W=\left\{w_{1}, w_{2}, \cdots, w_{t}\right\} \cup\{u\}$ be a subset of $V\left(G_{1}\right)$. Therefore, we can say that $\mathrm{W}$ is a resolving set of $G_{1}$. Because any pair distinct $x, y$ that has the same distance to vertex $u$ is distinguished by a vertex of $\left\{w_{1}, w_{2}, \cdots, w_{t}\right\}$. Whereas, any pair distinct that has different distance to vertex $\mathrm{u}$ can be distinguished by vertex $u$. Furthermore, we obtain $\operatorname{dim}\left(G_{1}\right) \leq t+1 \leq k$. In the same ways, we have $\operatorname{dim}\left(G_{2}\right) \leq k$.

The next Theorem 2 give the upper bound of the metric dimension of a bridge graph which is obtained from two connected graphs.

\section{Theorem2.}

Let $G_{1}$ and $G_{2}$ be two connected graphs and $u \in V\left(G_{1}\right)$, $v \in V\left(G_{2}\right)$. If $\operatorname{dim}\left(G_{1}\right)=t$ and $\operatorname{dim}\left(G_{2}\right)=s$, then $\operatorname{dim}\left(B\left(G_{1}, G_{2}, u v\right)\right) \leq t+s$

\section{Proof}

Let $W_{1}=\left\{w_{1}, w_{2}, \cdots, w_{t}\right\}$ and $W_{2}=\left\{w_{1}^{\prime}, w_{2}^{\prime}, \cdots, w_{s}^{\prime}\right\}$ be a resolving partition of $G_{1}$ and $G_{2}$, respectively. Let $G=$ $B\left(G_{1}, G_{2}, u v\right)$ and $W=W_{1} \cup W_{2}$ be subset of $V(G)$. Let $w^{*}$ be a vertex in $W_{1}$ which has a minimum of $d\left(w_{i}, u\right)$ for any $w_{i} \in W_{1}$. We will show that $\mathrm{W}$ is a resolving set of $G$.

Let $x, y$ be two distinct vertices of $G$. If $x, y \in V\left(G_{1}\right)$, then consider the distance $x$ and $y$ to vertex $u$. If $d(x, u)=$ $d(y, u)$, then the vertices $x$ and $y$ cannot be distinguished by any vertex in $G_{2}$. Therefore, the vertices $x$ and $y$ can be only distinguished by the members of $W_{1}=\left\{w_{1}, w_{2}, \cdots, w_{t}\right\}$. If $d(x, u) \neq d(y, u)$, then the vertices $x, y$ are distinguished by a vertex $w^{\prime \prime}$. So, if we have any distinct pair $x, y$ in $G_{1}$ then $r(x \mid W) \neq r(y \mid W)$. In the same argument, if we have any distinct pair $x, y$ in $G_{2}$ then $r(x \mid W) \neq r(y \mid W)$.

Now if $x \in G_{1}$ and $y \in G_{2}$, then consider distance $x, y$ to $w *$.If $d\left(x, w^{*}\right) \neq d\left(y, w^{*}\right)$, then clearly the vertices $x$ and $y$ are distinguished by $w^{*}$. If $d\left(x, w^{*}\right)=d\left(y, w^{*}\right)$, then consider shortest $L$ from $x$ to $w^{*}$. If $u \in L$ then $d\left(x, w^{*}\right)=$ $d(x, u)+d\left(u, w^{*}\right)$. Whereas, we have $d\left(y, w^{*}\right)=$ $d(y, u)+d\left(u, w^{*}\right)$. Since $d\left(x, w^{*}\right)=d\left(y, w^{*}\right)$, we obtain $d(x, u)=d(y, u)$. Since $u v$ is a bride edge, this implies $d(x, v)>d(y, v)$. So, we have $d\left(x, w^{\prime \prime}\right)>d\left(y, w^{\prime \prime}\right)$. Furthermore, we obtain $r(x \mid W) \neq r(y \mid W)$.

If $u \notin L$ then $d(x, u)+d\left(u, w^{*}\right)>d\left(x, w^{*}\right)$. Since $d\left(x, w^{*}\right)=d\left(y, w^{*}\right)$, we obtain $d(x, u)+d\left(u, w^{*}\right)>$ $d\left(y, w^{*}\right)=d(y, u)+d\left(u, w^{*}\right)$. So, we have $d(x, u)>$ $d(y, u)$. Therefore, $d(x, v)>d(y, v)$ and we have $d\left(x, w^{\prime \prime}\right)>d\left(y, w^{\prime \prime}\right)$. Furthermore, we obtain $r(x \mid W) \neq$ $r(y \mid W)$. These imply that $W$ is a resolving set of $G$.

For the simple example, if we have $G_{1}=P_{n}$ and $G_{2}=$ $P_{m}$, then consider the Figure 1 . 


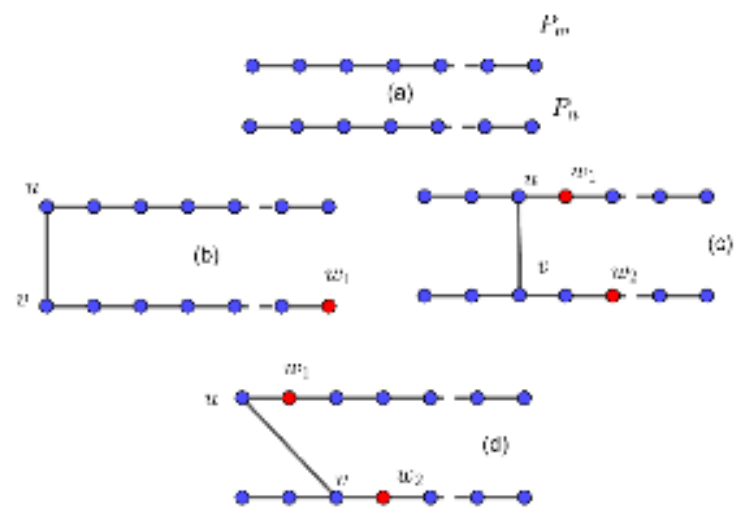

Fig. 1. (a) $P_{n}$ and $P_{m}$ are two path graph, (b) $G=B\left(P_{n}, P_{m}, u v\right)$ with $\operatorname{dim}(G)=1$, (c,d) $G=B\left(P_{n}, P_{m}, u v\right)$ with $\operatorname{dim}(G)=2$

In the Figure 1. We have the metric dimension of $G=$ $B\left(P_{m}, P_{n}, u v\right), \operatorname{dim}(G) \leq 2$.

The metric dimension of bridge graph from a homogeneous caterpillar and cycle graph

In this section, we show the metric dimensions of homogeneous caterpillar and cycle graphs. A homogenous caterpillar graph, $C(m, n)$ is a graph obtained by attaching $n$ leaves to each vertex $v_{i}$ of a path $P_{m}$. The path $P_{m}$ of $C(m, n)$ is called a backbone of $C(m, n)$. All leaves attached to a vertex $v_{i}$ is labeled by $w_{i, 1}, w_{i, 2}, \cdots, w_{i, n}$ and all vertices of $P_{m}$ of $C(m, n)$ is labeled by $v_{1}, v_{2}, \cdots, v_{m}$. A cycle graph, $C_{k}$ with $k \geq 3$ is a graph with each vertex $u_{i}$ which has degree two. So, the vertices of cycle $C_{-} k$ is labeled by $u_{1}, u_{2}, \cdots, u_{k}$.

The metric dimensions shown in following Theorem 2 is the metric dimensions of the bridge graph $B\left(G_{1}, G_{2}, a b\right)$ of the homogeneous caterpillar graph $G_{1}=C(m, n)$ and cycle graph $C_{p}$, specifically on the bridge edge of a vertex $u_{i}$ of cycle $G_{2}$ to endpoint of the backbone of $G_{1}$.

\section{Theorem 3.}

Let $C_{m, n}$ be a homogeneous caterpillar graph and $C k_{p}$ be a cycle graph. If $a \in V\left(C_{m, n}\right), b \in V\left(C_{p}\right)$, then $\operatorname{dim}\left(B\left(C_{m, n}, C_{p}, a b\right)\right)=m(n-1)+1, \quad$ for $a=v_{t}, t \in$ $\{1,2, \cdots, m\}$.

\section{Proof}

Without loss of generality (wlog), let $b=u_{1}$ and let $G=$ $B\left(C(m, n), C_{p}, v_{t} u_{1}\right)$ for some $t \in[2, m-1]$.

First, we will show $\operatorname{dim}(G) \leq m(n-1)+1$. Let $W=$ $\left\{w_{1,1}, \ldots, w_{1,(n-1)}, w_{2,1}, \ldots, w_{2,(n-1)}, \ldots, w_{m, 1}, \ldots, w_{m,(n-1)}, u_{k}\right\}$. To show $\operatorname{dim}(G) \leq m(n-1)+1$, we will show that $\mathrm{W}$ is a basis of $G$. Supposed $x, y \in G$. We consider the proof in three cases:

Case 1. For both of $x$ and $y$ in $C(m, n)$, we consider $d\left(x, v_{t}\right)$ and $d\left(y, v_{t}\right)$. If $d\left(x, v_{t}\right) \neq d\left(y, v_{t}\right)$, then $x$ and $y$ are distinguished by $w_{t, 1}$. If $d\left(x, v_{t}\right)=d\left(y, v_{t}\right)$, then consider the vertices $x$ or $y$ as a leaf or a vertex of backbone of $C(m, n)$. If the vertices $x$ and $y$ are two vertices of backbone $C(m, n)$ then $x=v_{i}$ and $y=v_{j}$ for some $i \neq j \in$ $[2, m-1]$. Therefore, since a leaf $w_{i, 1}$ is adjacent to $v_{i}$, then the vertex $d\left(x, w_{i, 1}\right)=1$, whereas the distance from vertex $y$ to $w_{i, 1}, d\left(x, w_{i, 1}\right)>1$. So, this implies that the vertices $x, y$ are distinguished by $w_{i, 1}$. If the vertices $x$ and $y$ are two leaves of $C(m, n)$, then let $x=w_{i, 1}$ and $y=w_{j, 1}$ for some $i \neq j \in[2, m-1]$. Since $d\left(w_{i, 1}, w_{i, 2}\right)=2$ and $d\left(w_{i, 1}, w_{i, 2}\right)>2$, then the vertices $x, y$ are distinguished by $w_{i, 2}$. If the vertex $x$ is a leaf and $y$ is a vertex od backbone $C(m, n)$, then wlog let $x=w_{i, 1}$ and $y=v_{j}$ for some $i \neq j \in$ $[2, m-1]$. Since $d\left(v_{j}, w_{j, 1}\right)=1$ and $d\left(w_{i, 1}, w_{j, 1}\right)>1$, then the vertices $x, y$ are distinguished by $w_{j, 1}$. These imply $r(x \mid U)=r(y \mid U)$.

Case 2. For both of $x$ and $y$ in $C_{k}$. If $d\left(x, u_{k}\right) \neq$ $d\left(y, u_{k}\right)$, then clearly, vertices $x$ and $y$ are distinguished by $u_{k}$. If $d\left(x, u_{k}\right)=d\left(y, u_{k}\right)$, then we have $d\left(x, u_{1}\right) \neq$ $d\left(y, u_{1}\right)$. So the vertices $x$ and $y$ are distinguished by $w_{t, j}$ for some $j \in\{1,2, \cdots, n-1\}$.

Case 3. For $x \in V(C(m, n))$ and $y \in V\left(C_{k}\right)$, consider the distance $x$ and $y$ to vertex $u_{k}$. If $d\left(x, u_{k}\right)=d\left(y, u_{k}\right)$, then $d\left(x, v_{t}\right) \neq d\left(y, v_{t}\right)$. So, the vertices $x$ and $y$ are distinguished by $w_{t, 1}$. If $d\left(x, u_{k}\right) \neq d\left(y, u_{k}\right)$, then clearly, vertices $x$ and $y$ are distinguished by $u_{k}$. These imply $r(x \mid U)=r(y \mid U)$

Base on the cases 1,2 , and 3 , we obtain that $W$ is a resolving set of $G$ with $|W|=(m(n-1)+1)$. Therefore, we have

$$
\operatorname{dim}(G) \leq m(n-1)+1
$$

Next, we will show $\operatorname{dim}(G) \geq m(n-1)+1$.

For a contradiction, suppose there is a set $U$ as a basis of $G$ with $|U|<(m(n-1)+1)$.

Let $U=\left\{z_{1}, z_{2}, \cdots, z_{m(n-1)}\right\} \subset V(G)$ with $|U|=$ $(m(n-1))$.

If all vertices $z_{i}$ in $U$ are in the vertices of $V(C(m, n))$, then there are at least two vertices $u_{2}$ and $u_{k}$ which have the same distance to vertex $v_{t}$. So, the vertices $u_{2}$ and $u_{k}$ have the same distance to any vertex in $V(C(m, n))$. Therefore, we have $d\left(u_{2}, z_{i}\right)=d\left(u_{k}, z_{i}\right)$. This implies that $r\left(u_{2} \mid U\right)=$ $r\left(u_{k} \mid U\right)$, a contradiction to $U$ as a basis of $G$.

If there is a vertex of $z_{t} \in U$ for some $t \in[1, m(n-$ 1)] which is a vertex of $V\left(C_{k}\right)$, then there are at least one vertex $v_{k}$ which has two leaves $w_{k, 1}$ and $w_{k, 2}$ for some $k \in$ $[1, m]$ such that $w_{k, 1}$ and $w_{k, 2}$ are not in set $U$. This is caused by there are $m(n)$ leaves of $C(m, n)$ whereas there are only $m(n-1)-1$ vertex $C(m, n)$ in set $U$. This implies $r\left(w_{k, 1} \mid U\right)=r\left(w_{k, 2} \mid U\right)$, a contradiction to $U$ as basis of $G$. These consequences, we have

$$
\operatorname{dim}(G) \geq m(n-1)+1 \text {. }
$$

Bases on inequations (1) and (2), we obtain $\operatorname{dim}(G)=$ $m(n-1)+1$.

\section{Theorem 4.}

Let $C(m, n)$ be a homogen caterpillar graph and $C_{k}$ be a cycle graph. If $a \in V\left(C_{m, n}\right), b \in V\left(C_{k}\right)$, then 
$\operatorname{dim}\left(B\left(C(m, n), C_{p}, a b\right)\right)=m(n-1), \quad$ for $a=w_{t, 1}$, for some $t \in[1, m]$.

\section{Proof}

Without loss of generality (wlog), let $b=u_{1}$ and let $G=$ $B\left(C(m, n), C_{p}, w_{t, 1} u_{1}\right)$ for some $t \in[1, m]$.

First, we will show $\operatorname{dim}(G) \leq m(n-1)$. Let $W=$ $\left\{w_{1,1}, \ldots, w_{1,(n-1)}, \cdots, w_{t, 2}, \ldots, w_{t,(n-1)}, \ldots, w_{m, 1}, \ldots, w_{m,(n-1)}, u_{k}\right\}$ with $|W|=m(n-1)$. We note that the set $W$ is different to $W$ in Theorem 1 or 2 , because the vertex $w_{t, 1}$ is in $W$ of Theorem 1 or 2, whereas in Theorem 3, the vertex $w_{t, 1}$ is not in $W$. Suppose $x, y \in G$. We consider the proof in three cases:

Case 1. For both of $x$ and $y$ in $C(m, n)$, we consider $d\left(x, w_{t, 1}\right)$ and $d\left(y, w_{t, 1}\right)$. If $d\left(x, w_{t, 1}\right) \neq d\left(y, w_{t, 1}\right)$, then they have $d\left(x, w_{t, 2}\right) \neq d\left(y, w_{t, 2}\right)$. So, thw vertices $x$ and $y$ are distinguished by $w_{t, 2}$. If $\left(x, w_{t, 1}\right)=d\left(y, w_{t, 1}\right)$, then consider the vertices $x$ or $y$ as a leaf or a vertex of backbone $C(m, n)$. If the vertices $x$ and $y$ are two vertices of backbone $C(m, n)$ then $x=v_{i}$ and $y=v_{j}$ for some $i \neq j \in$ $[2, m-1]$. Therefore, since a leaf $w_{i, 1}$ is adjacent to $v_{i}$, then the vertex $d\left(x, w_{i, 1}\right)=1$, whereas the distance from vertex $y$ to $w_{i, 1}, d\left(x, w_{i, 1}\right)>1$. So, this implies that the vertices $x, y$ are distinguished by $w_{i, 1}$. If the vertices $x$ and $y$ are two leaves of $C(m, n)$, then wlog let $x=w_{i, 1}$ and $y=w_{j, 1}$ for some $i \neq j \in[2, m-1]$. Since $d\left(w_{i, 1}, w_{i, 2}\right)=2$ and $d\left(w_{i, 1}, w_{i, 2}\right)>2$, then the vertices $x, y$ are distinguished by $w_{i, 2}$. If the vertex $x$ is a leaf and $y$ is a vertex of backbone $C(m, n)$, then let $x=w_{i, 1}$ and $y=v_{j}$ for some $i \neq j \in[2, m-1]$. Since $d\left(v_{j}, w_{j, 1}\right)=1 \quad$ and $d\left(w_{i, 1}, w_{j, 1}\right)>1$, then the vertices $x, y$ are distinguished by $w_{j, 1}$. These imply $r(x \mid W) \neq r(y \mid W)$.

Case 2. For both of $x$ and $y$ in $C_{k}$. If $x, y$ have the different distance to vertex $u_{k}, d\left(x, u_{k}\right) \neq d\left(y, u_{k}\right)$, then the vertices $x$ and $y$ are distinguished by $u_{k}$. If the vertices $x, y$ have the same distance to vertex $u_{k}$, then $x$ and $y$ are distinguished by $w_{t, 2}$. These imply $r(x \mid W)=r(y \mid W)$.

Case 3. For $x \in C(m, n)$ and $y \in V\left(C_{p}\right)$, If $d\left(x, v_{t}\right) \neq$ $d\left(y, v_{t}\right)$, then they are distinguished by $w_{t, 2}$. If $x$ and $y$ have the same distance to vertex $v_{t}, d\left(x, v_{t}\right)=d\left(y, v_{t}\right)=r$, then we obtain $\quad d\left(x, a_{p}\right)=d\left(x, v_{t}\right)+d\left(v_{t}, w_{t, 1}\right)+$ $d\left(w_{t, 1}, u_{1}\right)+d\left(u_{1}, u_{k}\right)=r+3$. Whereas, we have $d\left(y, u_{k}\right) \leq r$. This implies that the vertices $x, y$ are distinguished by $u_{k}$. These imply $r(x \mid W)=r(y \mid W)$.

Base on the cases 1,2 , and 3 , we obtain that $W$ is a resolving set of $G$ with $|W|=(m(n-1)+1)$. Therefore, we have

$$
\operatorname{dim}(G) \leq m(n-1)
$$

Next, we will show $\operatorname{dim}(G) \geq m(n-1)$.

For a contradiction, suppose there is a set $U$ as a basis of $G$ with $|Z| \leq m(n-1)-1$.
Let $Z=\left\{z_{1}, z_{2}, \cdots, z_{m(n-1)-1}\right\} \subset V(G)$ with $|U|=$ $m(n-1)-1$. We know that there are $m(n)-1$ leaves $w_{i, j}$ where $1 \leq i \leq m, 1 \leq j \leq n$ and $m$ center in $C(m, n)$.

If any vertex $v_{i}$ is only adjacent to one leaf which is not in set $Z$ namely every vertex of $\left\{w_{-}(1, n), w_{-}(2, n), \cdots, w_{-}(m, n)\right\}$ is not in $\mathrm{Z}$, then we have vertices $u_{2}$ and $u_{k}$ have the same distance to any vertex in $V(C(m, n))$. So, we have $d\left(u_{2}, z_{1}\right)=d\left(u_{k}, z_{1}\right)$. This implies that $r\left(u_{2} \mid Z\right)=r\left(u_{k} \mid Z\right)$, a contradiction to $Z$ as a basis of $G$.

If there is a vertex of $z_{t} \in Z$ for some $t \in$ $[1, m(n-1)]$ which is not a leaf of $C(m, n)$, then there are at least two leaves $w_{k, j}$ for some $k \in[1, m]$, we say $w_{k, 1}$ and $w_{k, 2}$, which have the same distance any vertex in $G$. This implies $r\left(w_{k, 1} \mid Z\right)=r\left(w_{k, 2} \mid Z\right)$, a contradiction to $Z$ as basis of $G$. These consequences, we have

$$
\operatorname{dim}(G) \geq m(n-1)
$$

Bases on inequations (3) and (4), we obtain $\operatorname{dim}(G)=$ $m(n-1)$.

\section{CONCLUSION}

In this paper, we gave three properties of the metric dimension of some bridge graphs as follows:

1. For any bridge graph $G$ with uv as a bridge edge and $G_{1}$ and $G_{2}$ are two components of $\mathrm{G}$, we obtained that the upper bound of metric dimension of $G_{1}$ or $G_{2}$ is at most dimension metric of $\mathrm{G}$.

2. For $G_{1}$ and $G_{2}$ be two connected graph and $u \in V\left(G_{1}\right)$, $v \in V\left(G_{2}\right)$, we obtain that $\operatorname{dim}\left(B\left(G_{1}, G_{2}, u v\right)\right)$ is at most the sum of metric dimension of $G_{1}$ and $G_{2}$.

3. For the homogeneous caterpillar graph $C_{m, n}$ and cycle graph $C_{p}$, we obtained the exact value of metric dimension of the bridge graphs $B\left(C_{m, n}, C_{p}, a b\right)$ where $a \in V\left(C_{m, n}\right), b \in V\left(C_{p}\right)$, namely

a. $\operatorname{dim}\left(B\left(C_{m, n}, C_{p}, a b\right)\right)=m(n-1)+1$, for $a=v_{t}$, and $t \in\{1,2, \cdots, m\}$.

b. $\operatorname{dim}\left(B\left(C(m, n), C_{p}, a b\right)\right)=m(n-1), \quad$ for $a=$ $w_{t, 1}$, for $t \in\{1,2, \cdots, m\}$.

\section{Acknowledgments}

This research was supported the research grants PNBP and Penelitian Dasar Unggulan Perguruan Tinggi, Ristek Dikti.

\section{REFERENCES}

[1] G. Chartrand, L. Eroh, M. A. Johnson, and O. R. Oellermann, "Resolvability in graphs and the metric dimension of a graph," Discret. Appl. Math., 2000, doi: 10.1016/S0166-218X(00)00198-0.

[2] G. Chartrand, E. Salehi, and P. Zhang, "The partition dimension of a graph," Aequationes Math., 2000, doi: 10.1007/PL00000127.

[3] S. Khuller, B. Raghavachari, and A. Rosenfeld, "Landmarks in 
graphs," Discret. Appl. Math., 1996, doi: 10.1016/0166218X(95)00106-2.

[4] B. Sooryanarayana, S. Kunikullaya, and N. N. Swamy, "Metric dimension of generalized wheels," Arab J. Math. Sci., 2019, doi: 10.1016/j.ajmsc.2019.04.002.

[5] I. G. Yero, D. Kuziak, and J. A. Rodríguez-Velázquez, "On the metric dimension of corona product graphs," Comput. Math. with Appl., 2011, doi: 10.1016/j.camwa.2011.03.046.

[6] M. Bača, E. T. Baskoro, A. N. M. Salman, S. W. Saputro, and D. Suprijanto, "The metric dimension of regular bipartite graphs," Bull. Math. la Soc. des Sci. Math. Roum., 2011.

[7] S. W. Saputro et al., "The metric dimension of the lexicographic product of graphs," Discrete Math., 2013, doi: 10.1016/j.disc.2013.01.021.

[8] Amrullah, S. Azmi, H. Soeprianto, M. Turmuzi, and Y. S. Anwar, "The partition dimension of subdivision graph on the star," in Journal of Physics: Conference Series, 2019, doi: 10.1088/17426596/1280/2/022037.

[9] Amrullah, E. T. Baskoro, S. Uttunggadewa, and R. Simanjuntak, "The partition dimension of subdivision of a graph," in AIP Conference Proceedings, 2016, doi: 10.1063/1.4940802.

[10] Amrullah, H. Assiyatun, E. T. Baskoro, S. Uttunggadewa, and R. Simanjuntak, "The partition dimension for a subdivision of homogeneous caterpillars,” AKCE Int. J. Graphs Comb., 2013.

[11] Amrullah, Darmaji, and E. T. Baskoro, "The Partition Dimension Of Homogeneous Firecrackers," Far East J. Appl. Math., vol. 90, no. 1, pp. 77-98, Mar. 2015, doi: 10.17654/FJAMJan2015_077_098.

[12] Amrullah, E. T. Baskoro, R. Simanjuntak, and S. Uttunggadewa, "The Partition Dimension of a Subdivision of a Complete Graph," in Procedia Computer Science, 2015, doi: 10.1016/j.procs.2015.12.075. 